\title{
ESTIMATION AND ANALYSIS OF URBAN TRAFFIC FLOW
}

\author{
Joonsoo Lee and Alan C. Bovik \\ The University of Texas at Austin \\ Department of Electrical and Computer Engineering \\ 1 University Station C0803, Austin TX - 78712-0240, USA
}

\begin{abstract}
This paper describes methods for extracting traffic flow information from urban traffic scenes. The ultimate goal is to collect a macroscopic view of traffic flow information in a fully automatic and segmentation-free way. First, traffic flow is calculated by optical flow estimation. Then, traffic flow regions are defined by the initial traffic flow, and further analysis is performed only in the defined traffic flow regions.

Basic statistics of the traffic flow vectors are studied. It is shown that traffic flow computed by optical flow estimation effectively captures traffic scene activity. Also, the statistics of traffic flow vectors contain meaningful and interesting characteristics. An example application demonstrates the applicability and potential uses of the statistics.
\end{abstract}

Index Terms - Traffic flow, optical flow, flow vector statistics, traffic surveillance

\section{INTRODUCTION}

There has been a great deal of work toward various surveillance applications in the field of image processing and computer vision [1]. Researchers in the area of intelligent transportation systems (ITS) have been greatly interested in various traffic surveillance applications. They have developed visual surveillance systems that can prevent traffic incidents, and enhance traffic flow in both urban and highway traffic scenes. Previous work on traffic surveillance systems usually focused on automating general surveillance tasks that used to be performed by human operators. However, recent traffic surveillance systems target more complicated and high-level traffic tasks, one of which is to collect urban traffic flow information for adaptive traffic control (ATC). The information collection task is currently performed by magnetometer sensors [2]. Since video cameras are already widely deployed and have a number of advantages over the magnetometer sensors, it is natural to consider the use of vision-based systems for collecting traffic flow information.

Most conventional traffic surveillance systems have utilized motion detection algorithms to detect each distinct moving vehicle $[3,4,5,6]$. Motion detection algorithms have been actively researched mainly to improve their accuracy and ro- bustness against noisy trivial motions, illumination changes, and occlusion. Once moving vehicles are well detected, each vehicle can be tracked and have trajectory information collected, which can be combined into an overall description of traffic flow. This procedure seems natural since it follows the prevalent procedure of conventional traffic surveillance systems: detection, tracking, and analysis.

However, we decide to take a different approach: collect traffic flow information directly, without detection of single moving objects. There are several advantages to this approach. First, in this approach, trajectory information for a single vehicle is not required, which is non-trivial to transform into the overall traffic flow descriptors. Secondly, motion detection is inherently an image-segmentation problem, which is exceedingly difficult to generally solve, thus efforts to improve it will be saved by skipping the motion detection step.

Our approach to collect traffic flow information requires computing optical flow from traffic videos. We decide to use optical flow because the output of optical flow computation provides a rough overview of traffic flow in the scene. Our ultimate goal of this research is to discover how to process raw flow information into useful and meaningful traffic flow descriptors effectively and automatically. This includes finding traffic flow regions, learning the statistics of traffic flow, analyzing traffic flow with prior statistics, and so on. Similar approaches were taken in [7, 8] for crowd flow analysis, in that both used optical flow estimation without explicit detection of moving objects. [8] sees human crowd as a set of particles, and this work was further extended to track individuals in high density crowd [9]. However, [7] and [8] deal with human crowd scenes which contain less regular flow pattern than traffic scenes. Though a similar approach was taken with traffic scenes [10], our work differs in that our goal is to provide holistic information of a traffic scene, while [10] yields local information of wrong activities inside a scene.

In this paper, we study optical flow estimation algorithms, detect traffic flow regions from a traffic video, learn the basic statistics of traffic flow, and perform a simple experiment with the learned statistics. 


\section{OPTICAL FLOW ESTIMATION}

Optical flow has been used in conventional video surveillance systems to detect motion [3], but the purpose of using optical flow in such systems is just to detect moving objects. However, the purpose of using optical flow in our system is to provide statistical traffic flow information. The role of optical flow in our system is essential and critical to final performance. Thus, application-specific assumptions must be considered to choose an algorithm for optical flow estimation from among the many available optical flow algorithms. For instance, speed and computational complexity was a major factor in selecting an optical flow algorithm in [3], and a variation of a region-based matching algorithm was selected because it was able to be implemented in real-time. Since the accuracy and robustness is a more important qualification for an optical flow algorithm in our system which may analyze flow over long time periods, we seek an optical flow algorithm that gives high accuracy and robustness in most traffic scenes.

A variety of optical flow algorithms, with their strengths and weaknesses, are presented in [11]. We performed experiments to find the most accurate and robust optical flow algorithms against typical traffic scenes, and we found the optical flow algorithm introduced by Black and Anandan in [12] to be well-qualified for our system. This algorithm produces dense smooth optical flow at motion boundaries where there exist multiple motions. This smoothness offers advantages over other optical flow algorithms for handling complex urban traffic scenes. The computational complexity of the algorithm is of reduced importance in this approach. The essence of this algorithm is to reformulate the least-squares estimation problem inside optical flow estimation problems by using a robust estimation technique. Since the robust estimation technique is less sensitive to outliers, it reduces errors at motion boundaries and improve robustness of the optical flow estimation. The theoretical details are presented in [12]. We used code distributed by the author, which applies the robust estimation technique to a gradient-based optical flow algorithm.

\section{TRAFFIC FLOW REGION DETECTION}

As a preliminary step for traffic flow analysis, we define areas where traffic flow is expected, which we call traffic flow regions. This is an important step because further analysis will be performed only in the defined traffic regions. The traffic flow regions can be learned and refined over time. Since we assume that traffic videos are taken from fixed cameras, the geometry of the video scene does not significantly change over time. Therefore, if we provide a sufficient amount of video with normal traffic activities as a training set, we will be easily able to define traffic flow regions. This process can be repeated periodically to reflect on-going variations of the traffic scene.
First, we compute optical flow at each pixel using the Black and Anandan algorithm. To exclude noisy optical flow, the optical flow at each pixel is averaged over time $L$. Instead of computing a simple average, we create further robustness by using a trimmed mean filter [13] to compute average optical flow vectors over a temporal window $L$, which effectively removes noisy optical flow estimates. The temporal window $L$ can be adjusted according to the frame rate of the video and the average expected speed of vehicles in the scene. We simply used $L=24$ here, which is equal to the frame rate of the video. We roughly define it as the average flow at each pixel, and this value is used to define traffic flow regions. If the magnitude of the average flow is greater than a threshold, the pixel is included in traffic flow regions. This process puts equal weights on all the flows greater than the threshold, regardless of the magnitude. It is based on the assumption that there should be a meaningful amount of flow in a given direction to be a part of traffic flow regions. The final output is a normalized occurrence of a flow region on each pixel (Fig. 1). Brightness means more occurrences, while darkness means fewer. If the regions must be represented in a binary format, the final output can be thresholded to generate binary region maps. Also, the normalized occurrence for each pixel can be used as a prior probability for future analysis. As shown in Fig. 1, the traffic flow regions are effectively able to represent actual traffic lanes and separate them.

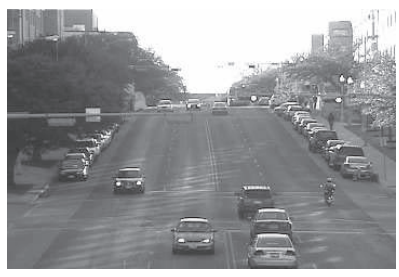

(a) Traffic scene image 1

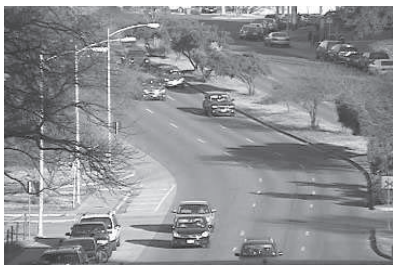

(c) Traffic scene image 2

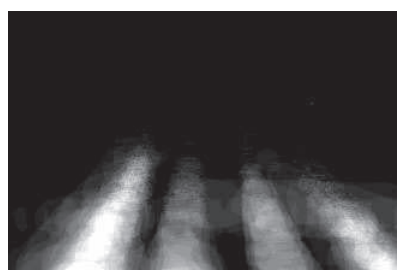

(b) Traffic flow region of (a)

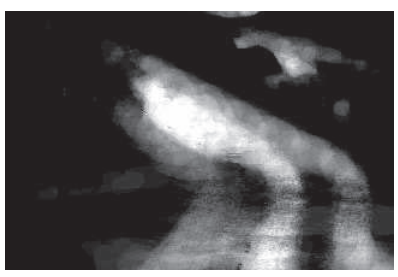

(d) Traffic flow region of (c)
Fig. 1. Defined traffic flow region

\section{STATISTICAL ANALYSIS OF TRAFFIC FLOW}

The ultimate goal of this research is to extract meaningful traffic flow information from traffic video streams that can be utilized in an adaptive traffic control system. To that end, we explore the statistics of traffic flow vectors since spatiotemporal modeling of traffic flow vectors will greatly benefit 
from the underlying statistics. There have been approaches to find the prior statistics of optical flow $[14,15]$, most have aimed to improve the accuracy of optical flow estimation. While [14] proposes a probabilistic formulation of the optical flow vectors, [15] provides detailed statistics of optical flow vectors computed from natural scene videos. In [15], the distributions of optical flow vectors and its derivatives are studied. The authors also formulate a Markov random field (MRF) prior revealing richer spatial statistics in larger neighborhoods by utilizing a Field-of-Experts (FoE) model. In short, the distribution of optical flow vectors shows a behavior similar to a Laplacian distribution, while the derivatives are heavy-tailed and resemble Student t-distributions. Since our videos are specifically targeting traffic scenes, the distributions are likely different from those on more general natural scenes in [15]. For instance, the distributions of traffic flow vectors are not zero-mean, and the distributions for horizontal and vertical vectors are not independent.

There are two different considerations when computing distributions of traffic flow vectors relative to other statistical studies on optical flow vectors. First, we only consider the traffic flow vectors inside the traffic flow regions defined, because only those vectors are of interest. Secondly, instead of dividing the vectors into vertical and horizontal components, we divide them into parallel and perpendicular to average flow, which is computed when traffic regions are defined,( because we are interested in the statistics of traffic flow vectors along traffic lanes. The direction of traffic lanes do not necessarily correspond to the vertical or horizontal directions of the video. As in [15], we compute the histogram of traffic flow vectors and the derivatives of traffic flow vectors and use them as scene-specific prior information of traffic flow.

\section{EXPERIMENTAL RESULTS}

\subsection{Traffic Video Collection}

Since there are not many publicly available videos on urban traffic scenes, we collected a number of traffic video streams near the University of Texas at Austin campus and used them for our experiments. The videos were taken at the center of roads, so they contain both sides of the roads. Each video is long enough to be divided into training and testing parts. The videos are in an uncompressed format to simplify their processing.

\subsection{Results Analysis}

We notice a couple of important characteristics from the histograms of traffic flow vectors and their derivatives (Fig. 2 ). The results are mostly as expected in Sec. 4. Basically, the histograms of traffic flow vectors shows a similar behavior to a Laplacian distribution, and the derivatives have heavy tails, like Student t-distributions. In the histograms of traffic flow vectors, the vertical and horizontal components are not

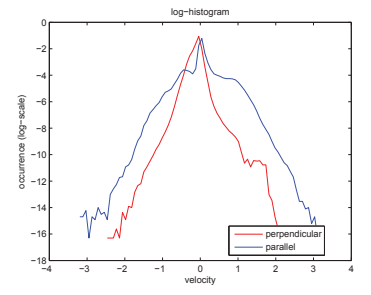

(a) Histogram of flow vectors

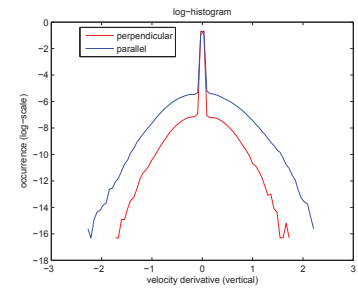

(c) Histogram of vertical derivatives

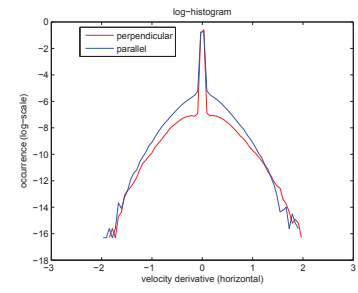

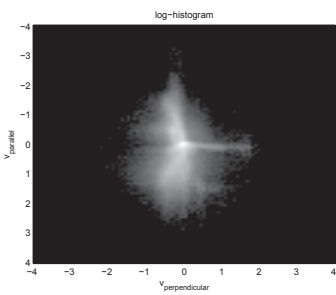

(b) Joint histogram of (a)

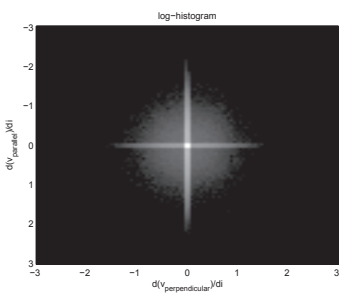

(d) Joint histogram of (c)

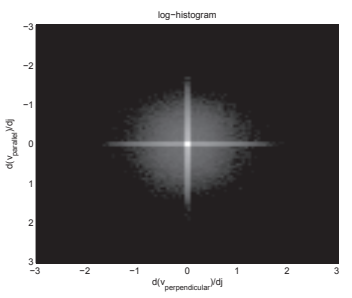

e) Histogram of horizontal derivatives(f) Joint histogram of (e)

Fig. 2. Histograms of flow vectors and derivatives

independent of each other, so the joint histogram with both components represents the statistics better than the two separate single histograms. One thing we notice is the similarity among the histograms of derivatives. Regardless of traffic scenes, they have nearly the same distribution, while the histograms of traffic flow vectors greatly depends on the traffic scenes. Therefore, we could use the histograms of traffic flow vectors for scene-specific purposes and the histograms of the derivatives for a validity test of estimated traffic flow.

\subsection{Flow Anomaly Detection}

Since the histograms of traffic flow vectors and their derivatives represent holistic traffic flows of a traffic scene, we can use them to detect anomalies of traffic flow at a certain time period. We can compute a histogram of traffic flow vectors for a short period time (1 second) and compare the histogram against the histogram previously learned for the specific traffic scene. We assume only normal activities are present in the scene during the learning period. We learned the statistics of traffic flow vectors over the first 1000 frames of a video and compared it to the later frames $\mathrm{o}$ the video. Among a number of different ways to compute the difference between two histograms, we chose to use 
the Kullback-Leibler (KL) divergence since it is a natural measure of difference between actual and desired probability distributions. Since anomalies seldom occur in actual traffic scenes, we devised a scenario to emphasize the effect of our method. We learned the statistics from a relatively quiet traffic scene and tested it against a sequence where the amount of overall traffic flow is small at the beginning and increases later. In Fig. 3, the result reflects expected traffic flow pattern, where the KL divergence grows as time goes. Larger KL divergence means flow pattern at the instant is more deviated from normal flow pattern, which we claim to be anomaly.

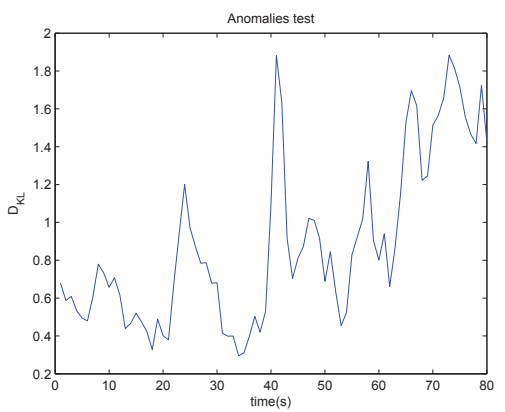

Fig. 3. Anomaly test with histograms

\section{CONCLUSION}

In this paper, a framework to collect traffic flow information from urban traffic scenes is proposed. As a first step, it shows a method to generate traffic flow by optical flow estimation. In order to take better advantage of the traffic flow, it presents ways of defining traffic regions, and of computing basic statistics of traffic flow vectors inside the traffic regions. It is shown that the characteristics of the statistics are different from those of general optical flow vectors, which can be utilized in further analysis of traffic flow. A simple problem of detecting anomalies in a traffic scene was provided as an application of traffic flow and its statistics.

\section{REFERENCES}

[1] W. Hu, T. Tan, L. Wang, and S. Maybank, "A survey on visual sruveillance of object motion and behaviors," IEEE Transactions on Systems, Man, and Cybernetics, vol. 34, no. 3, pp. 334-352, August 2004.

[2] M. Tubaishat, Y. Shang, and H. Shi, "Adaptive traffic light control with wireless sensor networks," in Proc. IEEE Consumer Communications and Networking Conference, January 2007, pp. 187-191.

[3] B. Maurin, O. Masoud, and N. P. Papanikolopoulos, "Tracking all traffic," IEEE Robotics and Automation Magazine, vol. 12, no. 1, pp. 29-36, March 2005.
[4] R. T. Collins et al., "A system for video surveillance and monitoring," Tech. Rep. CMU-RI-TR-00-12, Robotics Institute, Carnegie Mellon University, 2000.

[5] D. R. Magee, "Tracking multiple vehicles using foreground, background and motion models," Image and Vision Computing, vol. 22, no. 2, pp. 143-155, February 2004.

[6] A. Cavallaro, O. Steiger, and T. Ebrahimi, "Tracking video objects in cluttered background," IEEE Transactions on Circuits and Systems for Video Technology, vol. 15, no. 4, pp. 575-584, April 2005.

[7] E. L. Andrade, S. Blunsden, and R. B. Fisher, "Characterisation of optical flow anomalies in pedestrian traffic," in Proc. IEE International Symposium on Imaging for Crime Detection and Prevention, June 2005, pp. 73 78 .

[8] S. Ali and M. Shah, "A lagrangian particle dynamics approach for crowd flow segmentation and stability analysis," in Proc. IEEE International Conference on Computer Vision and Pattern REcognition, June 2007, pp. $1-6$.

[9] S. Ali and M. Shah, "Floor fields for tracking in high density crowd scenes," in Proc. European Conference on Computer Vision, October 2008, pp. 1-14.

[10] G. Monteiro, M. Ribeiro, J. Marcos, and J. Batista, "Wrong way drivers detection based on optical flow," in Proc. IEEE International Conference on Image Processing, October 2007, pp. 141-144.

[11] J.L. Barron, D. J. Fleet, and S.S. Beauchemin, "Performance of optical flow techniques," International Journal of Computer Vision, vol. 12, no. 1, pp. 43-77, February 1994.

[12] M. J. Black and P. Anandan, "The robust estimation of multiple motions: parametric and piecewise-smooth flow fields," Computer Vision and Image Understanding, vol. 63, no. 1, pp. 75-104, January 1996.

[13] A. C. Bovik, T. S. Huang, and D. C. Munson, "A generalization of median filtering using linear combinations of order statistics," IEEE Trans. Acoustics, Speech, Signal Process., vol. 31, no. 6, pp. 1342-1350, December 1983.

[14] E. P. Simoncelli, E. H. Adelson, and D. J. Heeger, "Probability distributions of optical flow," in Proc. IEEE Conference on Computer Vision and Pattern Recognition, June 1991, pp. 310-315.

[15] S. Roth and M. J. Black, "On the spatial statistics of optical flow," International Journal of Computer Vision, vol. 74, no. 1, pp. 33-50, August 2007. 